\title{
Activities of the AAPS In Vitro Release and Dissolution Testing Focus Group
}

\author{
Kailas Thakker ${ }^{1}$ and Vivian Gray ${ }^{2}$ \\ 'Analytical Solutions, Durham, NC. \\ ${ }^{2} V$. A. Gray Consulting, Hockessin, DE
}

\section{Face-to-Face Meeting}

he second IVR DT focus group face-to-face meeting was held on April $24^{\text {th }}$ in Research Triangle Park, North Carolina. About 40 people met in an offsite conference room facility hosted by GSK. The main topic of discussion was "Value of Dissolution throughout Development."The chain of the focus group, Qingxi Wang, opened the meeting and moderated throughout.

\section{Value of Dissolution throughout Development- Phase 1}

\section{Moderated by Stephen Mayock of Cardinal Health}

During Phase 1, dissolution medium selection is based on the characteristics of the API such as the solubility, $\mathrm{pK}_{\mathrm{a}}$ particle size range and distribution, and salt or amorphous form. The dissolution method developed during Phase 1 is for formulation development. The goal is to understand the dissolution characteristics of the API. Intrinsic dissolution of the API is generally determined and is performed using micro dissolution apparatus and fiber optics in combination with Apparatus 4 or other specific methods.

There was some discussion on BCS classification. During Phase 1 , it is not always possible to have enough in vivo data to make a relevant comparison. Therefore, computer modeling needs to be done. For BCS Class I drugs, it is possible to get an IVIVC from computer modeling using $\mathrm{pK}_{\mathrm{a}}$ data. It should be possible to use disintegration.

Many companies first develop a dissolution method for formulation development and supporting IVIVR and then change over to QC later.

For IVIVR/C correlation, the use of animal data is somewhat misleading, since animal data may not have any relevance to human data. It would be desirable to get human data sooner. Animal data can be used to generate rank-order.

\section{Value of Dissolution throughout Development- Phase 2 \\ Moderated by Ruben Lozano of BMS}

A case study of glyburide formulations was presented. Here, dissolution in bio-relevant media did not show any difference, but clinically there was significant difference. When API characteristics were used, a good predictive model was obtained. Introducing a $\mathrm{pH}$ gradient in the dissolution test gave a useful model.
Another case study was presented where the critical parameters were determined to be the mean particle size and particle size distribution. Different particle sizes and distributions gave different profiles. This information was useful in selecting a formulation. Therefore, in order to control and affect the performance of the formulation, particle size needed to be controlled.

Phase 2 is when one needs to look at IVIVC, but sometimes it may not be possible to have IVIVC. In that case, one should make formulations with different profiles, use alcohol in dissolution medium for poorly soluble drugs. One should also take a critical look at the API solubility at different $\mathrm{pH}$ values. Dissolution at this stage can still be used for formulation optimization.

Manufacturers are using tests such as disintegration and capsule rupture time as surrogates for the dissolution test. For Class 1 drugs in immediate-release formulations, the disintegration test is used. This can be achieved if there are other ways to evaluate stability.

Disintegration in vivo and in vitro have been compared by at least one company. They looked at radio-labeled dosage forms in vivo and found that in vivo disintegration was significantly different from in vitro disintegration.

For fast-dissolving tablets (> 90\% in $30 \mathrm{~min}$ ), rupture of the tablet is representative of dissolution, and one can use disintegration as a performance test. For a slow-dissolving formulation, one must determine the critical property that controls dissolution and use that as the performance test (e.g., erosion).

In case of oil-filled capsules, if the oil stays floating on top, capsule opening would be sufficient; if the contents go into solution, a dissolution test must be developed.

For extended-release formulations, a correlation of dissolution-release profile with performance profile is needed. It is necessary to understand the parameter that controls the release of API. Once that is known or understood, the formulator can design formulations that can give predictable release of API.

Dissolution has a lot of value prior to IVIVC. It gives insight into interactions of different parameters and allows synergistic effects that other surrogate test may not pick up. Dissolution still is perhaps the best test to evaluate formulation changes over time on stability.

Dissolution tests can identify changes that individual physical parameters do not, such as hardness, particle size, or distribution. 
For drugs with poor solubility, if dissolution test does not confirm the differences seen in vivo, it does not necessarily mean there is no IVIVC; it may be that the dissolution test needs to be revised.

With more and more drive toward fewer in vivo studies, it looks as if we should move early on toward one well-designed study that uses a relevant, predictive method and that would avoid failure at later stage. For example, one can pick three formulations based on dissolution in bio-relevant media and study them in vivo with reasonable certainty that there would be no failures.

\section{Value of Dissolution throughout Development-} Phase 3

\section{Moderated by Charles Tong of Pfizer, Inc.}

Dissolution data should allow one to obtain a

Bio-waiver. It is easy to get a Bio-waiver for BCS Class 1 drugs using dissolution.

In Phase 3, the dissolution test is more of a QC test whether there is correlation or not.

It is possible to use a QbD initiative to minimize $\mathrm{QC}$ testing. If another test, such as near IR, can be used to demonstrate blend homogeneity, then that test can be used to evaluate the impact of using another blender. More than one method is needed for evaluating changes when one has a complex relevant method. For example, dissolution in $\mathrm{pH} 6.8$ buffer was a QC method. But dissolution in $\mathrm{pH} 4.5$ buffer had rank-order correlation but only $65 \%$ release, thus it could not be used for a QC test but could be used anytime relevant data is needed. In the future, low release will be acceptable for QC.

Based on increased knowledge about the product and processes, it is possible to use new or alternate technologies.

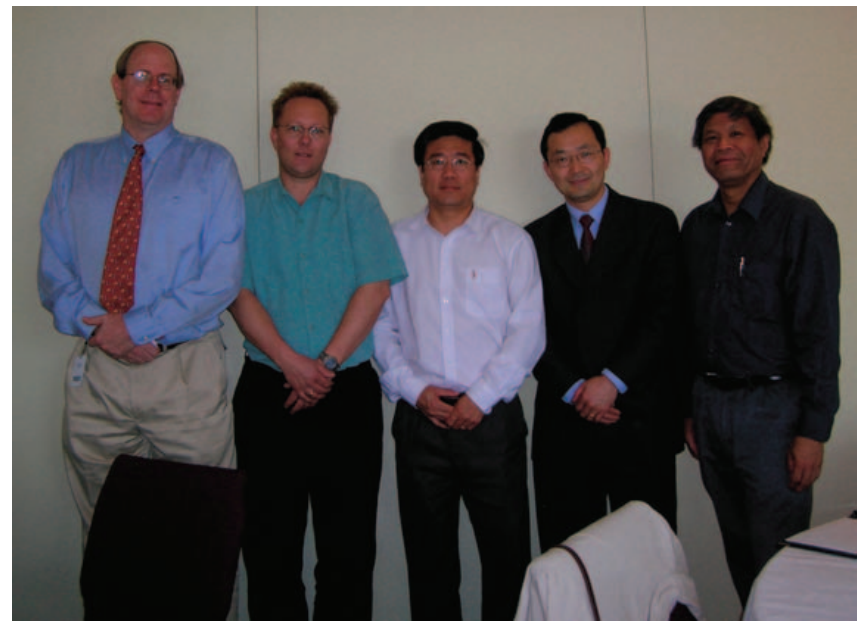

Discussion leaders:

Steve Mayock, Raimar Loebenberg, Qingxi Wang, Charles Tong, Ruben Lozano
Discussions on Mechanical versus Chemical Calibration of USP Apparatus:

Moderated by Alger Salt of GSK

Key points:

1) ASTM has drafted new procedures. (Note:This procedure has now been approved.)

2) FDA has endorsed these, but NOT officially.

3) Everyone had heard about ASTM procedures for mechanical calibration.

FDA labs are using mechanical calibration already. According to some at FDA, the failure of chemical calibration is analyst-related. Therefore, they now use prednisone tablets for training purposes.

It must be recognized that the ASTM procedure is an alternate procedure, thus it should be validated against the chemical calibration.

FDA has challenged the use of tablets (calibrator) for a test that is used to evaluate dissolution of tablets. The counter argument is that the system suitability test for any instrument is also done with the same solutions (system) that it is used to analyze.

The mechanical calibration procedure does not address vibration, vessel concentricity, and vessel smoothness.

PhRMA will refrain from changing to a mechanical calibration procedure alone until a specification is set for vibration. It is necessary to measure vibration when the vessel is filled with medium. Vibration is specific for an instrument, location, and company. It will take a long time to evaluate the vibration effects accurately.

A recently performed study explains why the calibrator ranges are so wide. In 30 labs, the variance between labs was high but was low within each lab. For five analysts and six testers, the residual variance was less than $4 \%$.

The crux of the matter is that the method is probably the source of variability. There is no way to quantitatively measure the percentage of $\mathrm{O}_{2}$ present in the medium. Vessels, the sampling zone, and the filtering process also make a huge difference. Different testers react differently. If one uses different vessels, one gets different results. Ideally, one must determine the gas content at the beginning and at the end of the test. USP/Pharma will re-run the collaborative test to tighten up the range for calibrators. Different testers react differently to perturbations. Some testers are sensitive to wobble, some to vibration. There should be an SOP to address calibrator failures. Issues such as wobbly baskets and peeling paddles must be taken into account. The May/June article of Pharmaceutical Forum will address the proposed changes USP is considering, which may add more value to the test and are more useful to the industry.

FDA and other groups have proposed ASTM method E25-03. Those implementing this method are requested to contact Vivian Gray (Dissolution Technologies), Bryan Crist (Varian), or Alger Salt (GSK). 
Finally, there are many instruments out in the field, especially in other countries where calibration is not enforceable, that will not pass the calibrator test.

\section{Discussions about the Dissolution workshop 2008:}

Tahseen Mirza is the chair of the program committee. Tentative dates are in the spring of 2008. It will be most likely a 2-21/2 day workshop. FDA, USP, and Industry representatives will be sought. Topics will focus on Phase-based dissolution and include the hydrodynamics of dissolution and dissolution of extended-release products.

\section{AAPS/SFDA Conference in Hangzhou, China}

On July 2 and 3,2007, a jointly sponsored conference was held, with representatives of the SFDA (the Chinese FDA) and the IVRDT focus group giving lectures on the topic of dissolution. On the first day, members of the Focus Group that spoke were Qingxi Wang, Vivian Gray, and Kailas Thakker. On day two, Lawrence Xu gave a talk. The subjects covered were the generic approval process, the added value of the dissolution test utilizing the concepts of BCS, method development for novel dosage forms and semi-solids, and an overview of dissolution equipment

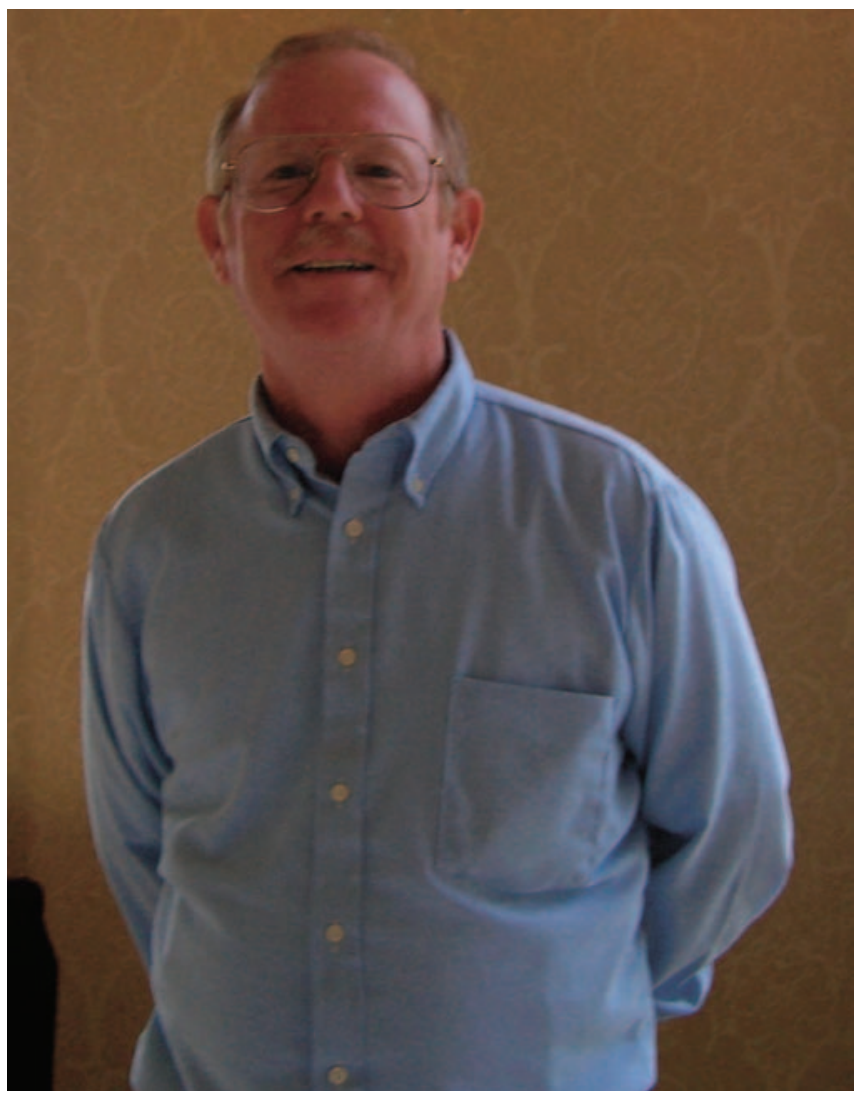

Organizer and Discussion leader: Alger Salt

Dissolution Technologies | AUGUST 2007 and sources of error. Qingxi Wang provided the translations.

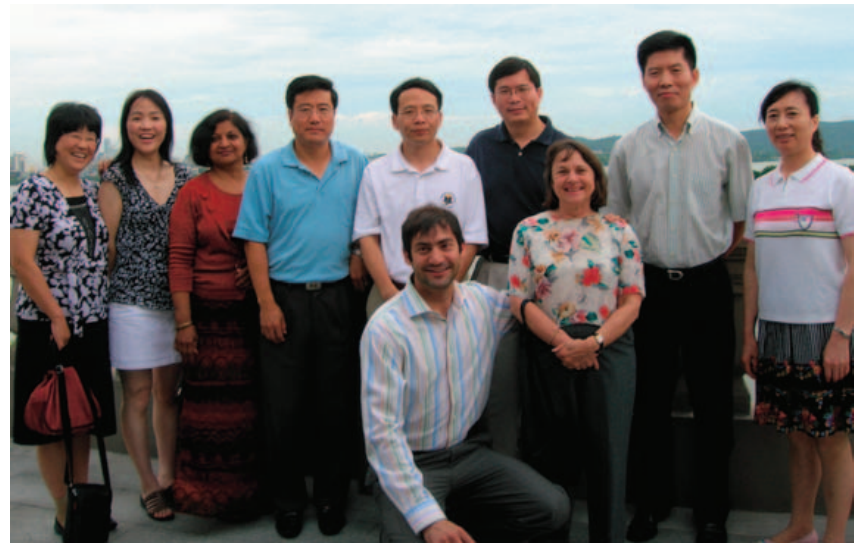

Conference Speakers: Liya Hong, Rose Gao, Kailas Thakker, Qingxi Wang, Lawrence Yu, Xiaoyi Guo, Shifei Chen, Huilan Li

Front now: Leonardo Allain, Vivian Gray

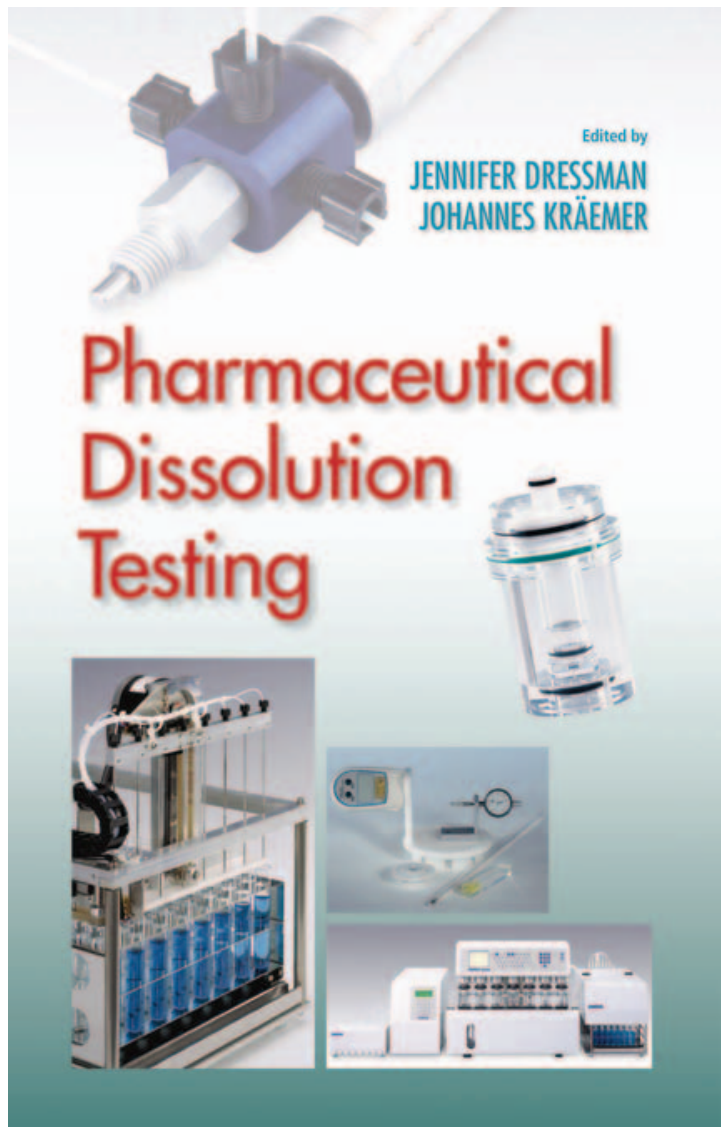

This book may be ordered online at the DT website at www.dissolutiontech.com. Price, including shipping, is $\$ 190.00$ for US and $\$ 210.00$ for outside USA. Credit card payment is available. 